\title{
ARASAAC: portal aragonés de la comunicación aumentativa y alternativa. Software, herramientas y materiales para la comunicación e inclusión
}

\section{ARASAAC: Aragonese portal of augmentative and alternative communi- cation. Software, tools and materials for communication and inclusion}

\begin{abstract}
Resumen: Ante las barreras para la comunicación que presentan personas con diferentes tipos de discapacidad, los Sistemas Aumentativos y Alternativos de Comunicación (SAACs) se convierten en una opción imprescindible para que puedan interactuar y modificar su entorno inmediato, a la vez que participar en el mundo que les rodea. En muchas ocasiones, es necesaria la utilización de diferentes productos de apoyo que faciliten estas acciones. Desde hace varios años, en Aragón, se han ido desarrollando, en colaboración con la Universidad de Zaragoza, diferentes ayudas técnicas y aplicaciones para la comunicación. Estos recursos requerían de pictogramas de libre distribución que complementaran su funcionalidad. El Gobierno de Aragón apoyó, desde el primer momento, esta línea de investigación e innovación en su creencia de que la comunicación es un derecho para todas las personas, independientemente de sus necesidades y posibilidades. ARASAAC nace bajo esta premisa con la colaboración del diseñador Sergio Palao, el Centro Aragonés de Tecnologías para la Educación (CATEDU) y los profesionales del Colegio Público de Educación Especial Alborada de Zaragoza. A lo largo de estos años, ARASAAC se ha convertido en un SAAC reconocido internacionalmente, que sigue creciendo y rompiendo barreras comunicativas en diferentes ámbitos como el educativo, hospitalario, geriátrico, adaptación de documentos, accesibilidad de los medios de comunicación, turismo accesible o señaléctica. Palabras-clave: Arassac. Comunicación Aumentativa y Alternativa. Inclusión.
\end{abstract}

Abstract: People with different kinds of disabilities are faced with communication difficulties, the Augmentative and Alternative Systems of Communications (SAACs) become an essential option so that these people can interact and change their immediate context, as well as participate in the world that surrounds them. On many occasions, it is necessary to use different support products which make these actions possible. For the last several years, in Aragón, diverse technical supports and applications for communication are being developed, in collaboration with the University of Zaragoza. These resources require pictograms for free use to complement their functionalities. The Government of Aragón supported this line of investigation and innovation from the very start with the belief that communication is a right for all people, independently of their needs and possibilities. ARASAAC is born under this premise with the collaboration of the designer Sergio Palao, Centro Aragonés de Tecnologías para la Educación (CATEDU)) and the professionals of Colegio Público de Educación Especial Alborada, Zaragoza. Throughout these years, ARASAAC has become a SAAC with international prestige that continues to grow and break down communication barriers in different fields such as, hospitals, old people's homes, document adaptation, accessibility to communication media, accessible tourism and sign posting.

Keywords: Arassac. Augmentative and Alternative Communication. Inclusión.

RODRIGO, José Manuel; CORRAL, Marcos David Romero. ARASAAC: portal aragonés de la comunicación aumentativa y alternativa. Software, herramientas y materiales para la comunicación e inclusión. Informática na Educação: teoria e prática, Porto Alegre, v. 16, n. 2, p. 27-38, jul./dez. 2013.
José Manuel Marcos Rodrigo

Centro de Educación Especial Alborada

David Romero Corral

Centro Aragonés de Tecnologías para la Educación

(CATEDU)

\section{Origen del proyecto ARASAAC}

D esde hace varios años, centros de Educación Especial de Aragón participan en experiencias de innovación e investigación, junto con otras instituciones, para el diseño y elaboración de recursos y ayudas técnicas relacionadas con la comunicación y la autonomía personal y social de sus alumnos.

A través de esta colaboración, surgieron aplicaciones como el Proyecto TICO (www. proyectotico.es) y Proyecto Vocaliza (www. vocaliza.es), que requerían de la incorporación de un catálogo de pictogramas para complementar su funcionalidad inicial. Como la premisa principal de estas dos aplicaciones era la libre distribución, los pictogramas incorporados a ambas debían reunir las mismas características. 
Fruto de esta necesidad, el Centro Aragonés de Tecnologías para la Educación (CATEDU), con la financiación del Departamento de Ciencia, Tecnología y Universidad, actualmente, Departamento de Industria e Innovación del Gobierno de Aragón, puso en marcha un grupo de trabajo inicial formado por el diseñador Sergio Palao, asesores del propio CATEDU y profesionales del C.P.E.E Alborada (Zaragoza).

El objetivo inicial de este grupo de trabajo fue la creación de un banco de pictogramas, que sirviera de soporte e instrumento facilitador de los procesos de comunicación a aquellas personas que demandaran apoyo visual en sus procesos de interacción con el entorno, tanto en el ámbito de la discapacidad, hospitalario, geriátrico o intercultural.

Paralelamente, el propio departamento estableció como otro objetivo fundamental la difusión y el acceso universal a la comunidad educativa y a la sociedad, en general, de todo el trabajo realizado a través de un portal en Internet.

Posteriormente, fueron surgiendo nuevos objetivos como la extensión de los recursos ofrecidos por el portal a nuevos colectivos con dificultades para la comunicación, la creación de herramientas on-line que permitieran a los profesionales y a las familias la elaboración de sus propios recursos y la distribución y difusión de los materiales elaborados.

Desde un primer momento, el Portal Aragonés de la Comunicación Aumentativa y Alternativa - ARASAAC (http://arasaac. org ), ha ido respondiendo a estos objetivos $y$, en la actualidad, sigue creciendo y evolucionando, aunque conservando siempre la idea original de libre distribución.

Para dar cobertura legal, se optó por una licencia Creative Commons (BY-NC-SA) para todos los recursos contenidos en el mismo. Esta licencia permite la difusión en cualquier ámbito (educativo, sanitario, asistencial, publicaciones, etc.) de los recursos y materiales que ofrece el portal, siempre y cuando se cite al autor (de los materiales, de los pictogramas, vídeos o de las fotografías), la fuente de la que han sido obtenidos y no se haga un uso comercial de éstos o de las obras derivadas. Podemos afirmar, por tanto, que el volumen de personas beneficiadas por este planteamiento no tiene límites, ya que el lenguaje visual y pictográfico es universal y todos los recursos que se ofrecen son de libre uso.

Para mejorar el acceso al portal de todos los usuarios, el laboratorio de Usabilidad de WALQA (Huesca) realizó un estudio y emitió un informe, a partir del cual se rediseñó completamente el portal inicial ARASAAC y en la actualidad, cumple todas las normas de accesibilidad y y usabilidad.

Igualmente, siendo conscientes de que la comunicación es un derecho universal para todas las personas y que deberían beneficiarse de los recursos contenidos en el portal el mayor número de países posible, se ha realizado la traducción del portal a Inglés, Francés, Rumano, Portugués y Portugués de Brasil. En la actualidad se está en proceso de traducción a otros idiomas.

\section{Recursos del Portal ARASAAC en la actualidad}

\section{- Catálogos de recursos gráficos}

Frente a diversos sistemas pictográficos comerciales que limitan su universalización y suponen un coste adicional para los usuarios, 
el portal ofrece varios tipos de recursos relacionados con la comunicación aumentativa y alternativa (CAA).

A nivel de recursos gráficos, el portal ARASAAC ofrece cinco catálogos: pictogramas en color, pictogramas en blanco y negro, fotografías, videos en Lengua de Signos Española y un sistema novedoso de fotografías con los distintos pasos para el signado de una palabra concreta.

Sin duda, los catálogos más reconocidos y con mayor proyección, nacional e internacional, son el catálogo de pictogramas en color y el de pictogramas en blanco y negro. En la actualidad, el catálogo de pictogramas en color contiene cerca de 9000 pictogramas únicos que, asociados a sus respectivas acepciones hacen que el catálogo disponga de más 14000 pictogramas. El catálogo de pictogramas en blanco y negro dispone de más de 12000 pictogramas. Su número es algo menor, ya que la introducción de algunos pictogramas, como las banderas, no tienen sentido en este catálogo.

El portal dispone, además, de dos catálogos novedosos a nivel nacional, elaborados en colaboración con la Fundación ASZA AragónAccesible, que recogen a través de vídeos y fotografías, las traducciones del diccionario de ARASAAC a Lengua de Signos Española (LSE). Además de la traducción de las acepciones, existen vídeos con las definiciones en LSE. Actualmente son más de 4000 palabras las que ya tienen traducción a este sistema.

Otra de las aportaciones novedosas es que el diccionario de palabras en castellano, sobre el que sustenta el portal, se encuentra traducido a 15 idiomas: árabe, chino, rumano, ruso, polaco, búlgaro, inglés, francés, italiano, alemán, portugués, portugués de Brasil, euskera, gallego y catalán.
En la actualidad, se está traduciendo a otros idiomas.

Cabe destacar que, además de la traducción escrita de las palabras en cada uno de estos idiomas, se ha incorporado la locución en formato mp3 en once de ellos, además del castellano. Estas locuciones pueden ser escuchadas desde la propia web y descargadas a nuestro ordenador.

\section{- Herramientas on-line}

Adicionalmente, el portal ARASAAC incorpora varias herramientas on-line, con sus correspondientes manuales, como son el creador de animaciones, el creador de símbolos, el creador de frases, creador de tableros de comunicación, creador de calendarios y creador de horarios, que facilitan la creación y elaboración de diversos materiales a los profesionales y a la familias.

Recientemente se han añadido dos nuevas herramientas que permiten elaborar Bingos $y$ Juegos de la Oca de modo sencillo.

\section{- Zona de descargas}

En esta zona, se ponen a disposición de todos los usuarios del idioma castellano los paquetes completos de pictogramas, imágenes, vídeos y fotografías en LSE con todas las acepciones que actualmente tienen asociadas en ARASAAC con la pretensión de hacer más útil la elaboración de materiales desde el propio ordenador del usuario y sin la necesidad de estar conectado a Internet.

En la actualidad, también, se puede acceder a la descarga de los paquetes de pictogramas (color y blanco y negro) en diez idio- 
mas: inglés, francés, danés, alemán, italiano, portugués, portugués de Brasil, catalán, gallego y euskera.

\section{- Materiales elaborados por los usua- rios}

Una de las secciones más significativas del portal y el verdadero "motor" de ARASAAC es la sección de Materiales. Está compuesta por más de $\mathbf{1 0 0 0}$ fichas, que nos dan acceso a más de $\mathbf{2 0 0 0 0}$ materiales elaborados y compartidos por los propios usuarios del portal. Este hecho la convierte en una sección única y muy valiosa en el mundo de la CAA. Estos materiales se distribuyen, también, bajo licencia Creative Commons.

\section{- Software}

En este catálogo, se describen y se enlazan programas gratuitos, algunos de ellos desarrollados en colaboración con distintos departamentos de ingenieros del Centro Politécnico Superior de la Universidad de Zaragoza y otras aplicaciones en las que se ha realizado una labor de asesoramiento a los autores. En todos los casos, dichas aplicaciones utilizan pictogramas de ARASAAC.

Actualmente están catalogados 20 programas y aplicaciones entre las que destacan: AraWord, Vocaliza, TICO, AraBoard, PictogramAgenda, PictoSelector, In-TIC, Comunicador CPA, Messenger Visual, E-Mintza, etc.

\section{- Ejemplos de uso}

Bajo el paraguas de la licencia Creative Commons, los pictogramas de ARASAAC han traspasado rápidamente el ámbito educativo y se van extendiendo en otros ámbitos como el de la salud, la adaptación de documentos, la accesibilidad universal, el acceso al ocio y tiempo libre, la participación social, el acceso a Internet, accesibilidad en los medios de comunicación,

En este catálogo, se recogen distintos ejemplos clasificados por varios criterios. Estos ejemplos pretenden mostrar a la sociedad en general distintas formas de utilización de la comunicación accesible para que den un paso adelante en este tipo de iniciativas. Este apartado está en continuo crecimiento debido a la mayor sensibilidad de organismos e instituciones hacia los usuarios con dificultades en la comunicación funcional.

\section{- Comunicación aumentativa}

En esta sección, se pretende ampliar la información a los usuarios acerca del tema de la CAA. Para ello, contiene las siguientes subsecciones:

- Enlaces / Otras webs: Listado de webs nacionales e internacionales, blogs o portales de recursos relacionados con la educación especial y la CAA, en particular.

- Bibliografía: Listado de libros en lengua castellana o inglés acerca sobre tema de la CAA. 
3 Colaboración de ARASAAC en el diseño, desarrollo y testaje de programas de software relacionados con la CAA y asesoramiento a desarrolladores

El proyecto ARASAAC, desde el inicio, ha venido colaborando y asesorando a distintos proyectos de software que incluyen los pictogramas de ARASAAC para diferentes sistemas operativos (Windows y Linux), para dispositivos móviles e, incluso, para consolas portátiles (Nintendo DS).

Es muy importante destacar que los grandes avances que se están produciendo en los sistemas operativos para dispositivos móviles (iOs y Android), además del aumento de la oferta y demanda de este tipo de dispositivos, aumentan las expectativas de desarrollo de nuevas aplicaciones libres para la CAA, que pueden incorporar los recursos de ARASA$\mathrm{AC}$ a sus desarrollos.

El portal ARASAAC ha participado en el diseño, desarrollo y testaje de varios programas de software y ha aportado sus recursos para introducirlos dentro de otros. Entre ellos, podemos destacar Proyecto TICO, Proyecto Vocaliza, AraWord y AraBoard., los cuales vamos a describir brevemente.

- Proyecto TICO (www.proyectotico.es)

El Proyecto TICO - Tableros Interactivos de Comunicación consiste en una aplicación informática que permite generar y utilizar tableros de comunicación de forma interactiva. Además, incorpora varias opciones de barrido, por lo que está totalmente recomendada para ser utilizada por usuarios con limitaciones motrices graves.
Esta aplicación nace del acuerdo de colaboración entre el CPEE Alborada y el Departamento de Informática e Ingeniería de Sistemas del Centro Politécnico Superior (CPS) de la Universidad de Zaragoza.

- Proyecto Comunica (www.vocaliza.es)

El Proyecto Comunica, otra de las aplicaciones precursoras de ARASAAC, surge a partir de la colaboración del Grupo de Tecnologías de las Comunicaciones (GTC) del Instituto en Investigación en Ingeniería de Aragón (I3A) en la Universidad de Zaragoza con los profesionales del CPEE Alborada para poner al servicio de la comunidad educativa la investigación y desarrollo en Tecnologías del Habla. El proyecto Comunica consta de tres aplicaciones, basadas en el reconocimiento de voz y enfocadas al trabajo de los distintos niveles del lenguaje oral, partiendo desde la habilidades previas hasta el nivel pragmático del lenguaje. El proyecto nace con la intención de que todas las herramientas desarrolladas bajo su amparo sean de libre distribución.

- AraWord (www.proyectotico.es/wiki/index.php/AraWord)

AraWord es una aplicación informática, basada en la premisa de software libre, consistente en un procesador de textos para la generación de documentos en el ámbito de la comunicación aumentativa y alternativa que permite la escritura simultánea con texto y pictogramas.

A través de un menú sencillo e intuitivo, la herramienta aprovecha las posibilidades que ofrecen los recursos gráficos de ARASAAC para convertir, cualquier texto que escribamos, en un texto adaptado con pictogramas, de forma instantánea. 
- AraBoard (http://giga.cps.unizar.es/ affectivelab/araboard.html)

AraBoard es un conjunto de herramientas diseñadas para la comunicación alternativa y aumentativa, cuya finalidad es facilitar la comunicación funcional, mediante el uso de imágenes y pictogramas, a personas que presentan algún tipo de dificultad en este ámbito. Dada la versatilidad de estas herramientas, AraBoard también puede ser utilizado para crear tableros con rutinas sencillas $y$ tableros para anticipar la realización de cualquier tarea prevista. Esta aplicación ha sido desarrollada por el grupo GIGA y el grupo EINA de la Universidad de Zaragoza, en colaboración con el C.P.E.E. Alborada y el CATEDU.

AraBoard permite crear, editar y usar tableros de comunicación para distintos dispositivos (ordenador, smartphone o tablet), así como para distintos sistemas operativos.

Con AraBoard podemos crear tableros desde una hasta treinta y dos casillas, utilizando para ello las distintas combinaciones posibles: 1 fila $\times 2$ columnas, 2 filas $\times 2$ columnas, 3 filas $\times 4$ columnas, ..., 4 filas $\times 8$ columnas. Esta característica convierte también a la herramienta en una posible alternativa para personas con discapacidad motriz y necesidades comunicativas muy básicas.

ARASAAC también ha participado en el desarrollo de otros proyectos de software libre, nacionales e internacionales, asesorando a sus desarrolladores y facilitando todos los recursos disponibles en el portal.

En el marco de esta colaboración, podemos destacar la participación en programas de sotfware libre para PC, como PictoSelector (http://www.pictoselector.eu/), In-TIC (www. intic.udc.es), así como otros proyectos en desarrollo como el chat de mensajería instantá- nea mediante pictogramas de la Fundación El Maresme (http://www.messengervisual. com/) o el de la Universidad Politécnica de Madrid, que todavía se encuentra en desarroIlo.

En el ámbito de los dispositivos móviles (smartphones y tablets), podemos destacar la colaboración en diferentes aplicaciones relacionadas con la CAA:

- Comunicador CPA (http://www.comunicadorcpa.com/)

- PictogramAgenda(http://www.lorenzomoreno.com/index.php/es/ software/79-pictogramagenda)

- e-Mintza (http://fundacionorange.es/ emintza.html)

- Pictodroid (http://www.accegal.org/ pictodroid/)

- Proyecto Arcón (http://www.arconvoz.es/)

- AAC Speech Communicator (http:// aacspeech.org/)

- TTalk_AAC (http://www.t-box.mobi/)

- iTucan Talk (https://play.google.com/ store/apps/details?id=com. itucanworking)

El interés por introducir los pictogramas de ARASAAC se ha extendido también a programas comerciales como Communicate in Print 2, Symbols for Windows, Ablah, Baluh, Qué fala!,..., Con el fin de respetar las condiciones establecidas por la licencia CC (BY-NC-SA) la inclusión de los pictogramas se realiza a través de paquetes de importación que se proporcionan de modo gratuito y libre a cualquier usuario que desee descargarlo independientemente de si dispone el programa con licencia o simplemente dispone de una demo. 
Lo que no permite la licencia Creative Commons BY-NC-SA es que los pictogramas se incorporen en ningún software comercial de serie $y$, por tanto, haya que pagar por ellos en tanto estamos pagando una licencia para adquirir el software. Además, es necesario que, en el proceso de instalación posterior, se cite expresamente los términos de la licencia de los mismos.

\section{Ejemplos de uso y colaboración con organismos e instituciones en la mejora de la accesibilidad en la comunicación}

La relevancia del proyecto, a nivel nacional e internacional, se ve reflejada en el gran número de organismos, entidades, asociaciones, fundaciones, universidades y profesionales con Ios que ARASAAC colabora aportando los recursos del portal y asesorando en el desarrollo de sus proyectos.

En el ámbito editorial, la aparición de ARASAAC ha colaborado a implementar la accesibilidad a la comunicación en documentos y publicaciones periódicas. Actualmente, ARASAAC tiene presencia, entre otros, en documentos como la "Convención de Derechos de las Personas con Discapacidad" (CEAPAT) traducida a pictogramas de ARASAAC, el libro "Comunicación y Aumentativa. Guía de referencia" (Clara Delgado, CEAPAT), en "Mi comunicador de pictogramas" (CEAPAT), en la Guía "Defendemos nuestros derechos en el día a día" editado por FEAPS, en el "Plan de Acción para las personas con discapacidad" (FEAPS), en "Un lugar donde quiero vivir: Mi casa" (FEAPS Madrid) y en publicaciones periódicas como la Guía de Ocio Infantil y Familiar en Zaragoza "Menudas Ideas".
En lo referente a los medios de comunicación, ARASAAC ha logrado que se empiecen a apoyar iniciativas para lograr la accesibilidad a la información mediante la subtitulación de noticias con pictogramas.

ARASAAC colabora con el canal autonómico aragonés Aragón Radio $\mathbf{2}$ subtitulando noticias de la página web del citado canal con los pictogramas del portal. Cada día se subtitula una noticia de actualidad $y$, semanalmente, los alumnos del C.P.E.E. Alborada (Zaragoza) envían sus noticias redactadas por ellos mismos con pictogramas en los Talleres de Prensa que tienen programados en sus aulas. Fruto de esta colaboración ha sido la obtención del VII Premio Periodístico "Por la integración", que otorga el IMSERSO. ARASAAC también colabora con el periódico Heraldo de Aragón, subtitulando, cada semana, una noticia con pictogramas que aparece en la portada del Heraldo Escolar. Ambas experiencias pretenden lograr que las noticias sean más accesibles a todas las personas y concienciar de la necesidad de hacer extensible esta idea a otros medios de comunicación.

A nivel institucional, el Ayuntamiento de Málaga, a través del área de accesibilidad, se propuso como objetivo la señalización con pictogramas de ARASAAC de todas sus dependencias municipales, postes de información y rutas turísticas, haciendo accesibles todos estos servicios para los usuarios con dificultades comunicativas,. Así mismo, para que los servicios que ofrece también fueran accesibles, se han creado libros de comunicación con pictogramas para restaurantes, centros comerciales, taxis, etc., que facilitan la atención a todos los ciudadanos por igual. Finalmente, en las últimas elecciones municipales fueron pioneros al señalizar a través de pictogramas las mesas electo- 
rales y crear documentos en fácil lectura con apoyo de pictogramas para facilitar el derecho al voto.

Continuando en el ámbito de la señalética, otros ayuntamientos y concellos (Cambados, A Illa de Arousa y Ribadumia), a través de la Asociación "Por Dereito" han señalizado las dependencias municipales con pictogramas de ARASAAC.

Todas estas acciones han tenido una gran repercusión en la prensa local y regional de dichos ayuntamientos y concellos, lo que ha facilitado que varios ayuntamientos de la zona sigan el ejemplo y colaboren en la sensibilización y concienciación para que la comunicación sea accesible en todos los entornos.

En la actualidad, siguiendo la iniciativa, el Ayuntamiento de Zaragoza ha creado una mesa de trabajo con dotación económica para la señalización de todas las dependencias para personas con limitaciones cognitivas, en las que el equipo de ARASAAC colabora como asesor y facilita todos los recursos disponibles. La mesa acordó afrontar la señalización este año de 4 dependencias municipales e ir ampliando éstas en años sucesivos. Más concretamente, los espacios que se señalizarán este año son: CDM Palafox, biblioteca María Moliner, Centro de Historia y la Junta Municipal de Casco Histórico).

Con el fin de formalizar la colaboración de ARASAAC con el Ayuntamiento de Zaragoza, el día 3 de Enero de 2013 se firmó un convenio de colaboración entre el Gobierno de Aragón y el Ayuntamiento de Zaragoza por el que el consistorio usará los recursos de ARASAAC en la señalización de las dependencias municipales.

Otros centros dependientes del IMSERSO como el Centro de Rehabilitación de Minusválidos Físicos de Salamanca también han señalizado todas sus estancias con picto- gramas de ARASAAC.

El proyecto supuso la señalización de cinco plantas, incluyendo el sótano. La señalización se realizó por tipos de estancias y pisos. En cada una de las estancias se colocó una placa $y$, en cada una de ellas, se incluyó texto en alto contraste, texto en Braille y pictograma.

Otro ejemplo a tener en cuenta es de la Fundación Privada Àuria de Igualada, una entidad sin ánimo de lucro, especializada en la atención a personas adultas con discapacidad que ha señalizado todo el edificio, incluyendo dependencias, placas de información y ascensores. Las placas de las distintas dependencias incluyen texto, pictograma y alfabeto manual dactilológico. También se ha dejado un espacio para incluir el texto en braille.

En el campo de la investigación universitaria, ARASAAC mantiene una estrecha colaboración con la Unitat de Tècniques Augmentatives de Comunicació -UTAC (https://sites.google.com/site/utacub/) dependiente de la Universidad Autónoma de Barcelona para recibir asesoramiento, por parte de todo el equipo, sobre la conceptualización y esquematización más adecuada del vocabulario UTAC-CACE y la creación de un sistema pictográfico que sirva de base a usuarios de distintas franjas de edad y con un nivel cognitivo más alto.

En el campo de la salud se puede destacar colaboraciones con distintas intituciones como el CEAPAT, que han dado lugar a documento como el "Cuaderno de Apoyo a la Comunicación con el paciente" que, recientemente ha sido publicado y traducido a diferentes idiomas. Este Cuaderno se repartirá en hospitales y centros de salud con el objetivo de concienciar al personal sanitario en la necesidad de utilizar un sistema de comunicación aumenta- 
tiva con pacientes que, por distintas circunstancias, tengan dificultades para comunicarse.

\section{Difusión}

El portal ARASAAC, desde sus comienzos, es financiado en su totalidad por el Departamento de Industria e Innovación y por el Departamento de Educación, Universidad, Cultura y Deporte del Gobierno de Aragón. Por lo tanto, desde sus inicios, el portal no ha contado con patrocinadores externos que pudieran hacer una mayor publicidad de todos los eventos del mismo.

Han sido Internet y las propias redes sociales quienes han asumido esa labor de "publicidad" y difusión. La extensión del portal a través de páginas web de referencia en el mundo de las necesidades educativas especiales, los blogs de profesionales y familias, el alojamiento de videos en Youtube $y$, posteriormente, Twitter, Pinterest, Google+ $y$ Facebook han conseguido que sea conocido $y$ visitado por millones de personas al año. Muchos de ellos, en la actualidad, se han convertido en grandes colaboradores y difusores del portal y sus contenidos.

\section{Reconocimientos}

Entre los reconocimiento y premios más destacables podemos destacar:

\section{ARASAAC, PREMIO DE LA FEDERACIÓN AUTISMO MADRID 2013 POR SU TRABA- JO POR LA INCLUSIÓN DE LAS PERSONAS CON AUTISMO}

En el marco de la semana del Día Mundial de Concienciación sobre el Autismo, la Federación
Autismo Madrid ha otorgado en esta edición el Reconocimiento al trabajo por la Inclusión a ARASAAC, Portal Aragonés de la Comunicación Aumentativa y Alternativa, como ejemplo de trabajo en pro de los derechos de las personas con discapacidad en general y con TEA en especial. ARASAAC ha puesto a disposición de la sociedad un amplio catálogo de pictogramas de fácil manejo y comprensión, adaptados a la realidad social actual, que permiten mejorar la comunicación de estas personas gracias al surgimiento de gran número de aplicaciones gratuitas y accesibles. ARASAAC está contribuyendo en gran manera al derecho universal de la comunicación, facilitando el acceso a la sociedad, la cultura, la información y el ocio a las personas con TEA.

\section{ARASAAC, PREMIO CINE Y SALUD 2013}

Dentro de la Jornada Día Mundial de la Salud 2013 (y XI Certamen de Cortometrajes), ARASAAC recibió el Premio Cine y Salud 2013, en el ámbito aragonés.

\section{PREMIO ACCESIBILIDAD UNIVERSAL 2010}

ARASAAC fue galardonado con el primer premio en la categoría de Tecnologías de la Comunicación e Información. ARASAAC competía como finalista en esta categoría con otros dos candidatos: Pilar del Valle, por sus propuestas de diseño basadas en e-accesibilidad para soluciones en salud personal y Óscar Saz, por el desarrollo de herramientas de accesibilidad al ordenador e Internet basadas en la voz.

El objetivo de estos premios, que se celebran desde 1998, es fomentar la sensibilización social en la promoción de medidas encaminadas a la plena accesibilidad universal y la promoción de la autonomía personal. 


\section{PREMIO PERIODÍSTICO "POR LA INTEGRACIÓN" POR LA "SUBTITULACIÓN DE NOTICIAS DE ARAGÓN RADIO CON LOS PICTOGRAMAS DE ARASAAC"}

Aragón Radio, el CPEE Alborada y ARASAAC fue reconocido con el "VII Premio Periodismo... Por la integración", otorgado por el Centro de Recuperación de Personas con Discapacidad Física y/o Sensorial del Imserso en Salamanca. Este premio pretende reconocer los trabajos periodísticos y la importante labor que desarrollan los profesionales de la comunicación en favor de la integración social y laboral de las personas con discapacidad.

Con este premio se quiso destacar la labor de Aragón Radio por la "integración de personas con discapacidad en sus contenidos, por la participación de las mismas en la elaboración de noticias para el segundo canal, y por la inclusión de éstas mediante lenguaje aumentativo, adaptado y asequible para la comunicación con personas que presentan dificultades".

\section{FINALISTA DE LOS PREMIOS PRÍNCIPE DE VIANA DE ATENCIÓN A LA DEPENDEN- CIA 2010}

El Premio Internacional Príncipe de Viana de Atención a la Dependencia lo otorgan el Gobierno de Navarra y Caja Navarra para reconocer a las personas o entidades que hayan realizado investigaciones relevantes y prácticas innovadoras en el ámbito de la atención a las personas dependientes, en la promoción de la autonomía personal y en la prevención de las causas que provocan la dependencia.

De entre las 61 candidaturas que finalmente se presentaron, fue seleccionado el Centro Aragonés de Tecnologías para la Educación por la creación del Portal Aragonés de la Comuni- cación Aumentativa y Alternativa (ARASAAC). Los otros 6 finalistas fueron el profesor Jesús Vaquero Crespo, la deportista Teresa Silva Fernández, la Confederación Española de Organizaciones de Mayores que, finalmente, resultó ganadora; el doctor Jim Van Oos, el grupo musical Staff Benda Bilili y la Fundación Atena Psicoballet.

En el momento actual, estamos pendientes de dos nominaciones que todavía no se han determinado.

Por un lado, ARASAAC es Candidato a los Premios Aragoneses del año 2013, en el apartado de Ciencia y Tecnología.

El Portal Aragonés de la Comunicación Aumentativa y Alternativa ARASAAC ha sido seleccionado por el Periódico de Aragón, dentro del apartado de Ciencia y Tecnología, en la XX Edición de los Premios Aragoneses del Año, un certamen que reconoce los méritos personales y profesionales de ciudadanos y colectivos aragoneses que desarrollan su labor en diferentes ámbitos, siempre con trascendencia social.

Por otro lado, ARASAAC es CANDIDATO A LOS PREMIOS PRÍNCIPE DE ASTURIAS 2013 EN LA CATEGORIA DE COMUNICACIÓN Y HUMANIDADES

Los Premios Príncipe de Asturias están destinados a galardonar la labor científica, técnica, cultural, social y humana realizada por personas, instituciones, grupos de personas o de instituciones en el ámbito internacional y se conceden en ocho categorías diferentes: Artes, Letras, Ciencias Sociales, Comunicación y Humanidades, Investigación Científica y Técnica, Cooperación Internacional, Concordia y Deportes. ARASAAC ha sido seleccionado como candidato en la categoría de Comunicación y Humanidades. 


\section{Conclusión}

Tras estos seis años desde la aparición del portal, todo el equipo de ARASAAC, así como las familias, profesionales e instituciones que colaboran en este proyecto han asumido $y$ han hecho suya la idea que subyace debajo del mismo, que no es otra que "la comunicación es un derecho universal que todas las personas" y este derecho deben disfrutarlo en igualdad de condiciones, en tanto que facilita su acceso a la sociedad, la cultura, la información, el ocio, etc.

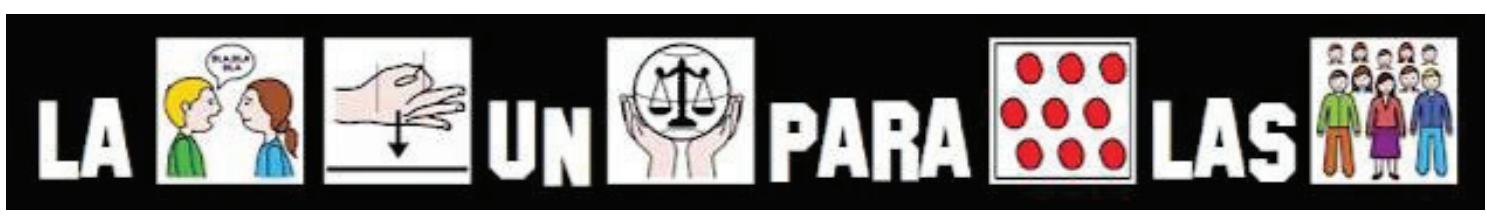

En esa aspiración, ARASAAC quiere ser parte activa, facilitando la comunicación a aquellas personas que requieren de sistemas pictográficos para la comunicación y defendiendo su inclusión en todos los ámbitos de la sociedad.

\section{Enlaces de interés}

\section{Páginas web de ARASAAC:}

http://arasaac.org/

http://www.catedu.es/arasaac/

\section{Blog:}

http://blog.arasaac.org/

\section{Facebook:}

http://www.facebook.com/pages/Arasaac-Portal-Aragonés-de-la-Comunicación-Aumentativa-y-Alternativa/326389010786376

\section{Twitter:}

http://twitter.com/arasaac 


\section{Pinterest:}

http://pinterest.com/arasaac/

\section{Google+:}

https://plus.google.com/u/0/100299612271876737156/posts

\section{Correo electrónico:}

arasaac@gmail.com

Submetido em 15 de agosto de 2013.

Aprovado em 04 de setembro de 2013.

José Manuel Marcos Rodrigo: Logopeda del Centro de Educación Especial Alborada de Zaragoza - Zaragora Espanha.

E-mail: arasaac@gmail.com

David Romero Corral: Asesor del Centro Aragonés de Tecnologías para la Educación (CATEDU) - Aragón - Espanha.

E-mail:dromero@educa.aragon.es 